\title{
Microglia Play an Essential Role in Synapse Development and Neuron Maturation in Tissue-Engineered Neural Tissues
}

\author{
Huimin Zhu', Xin Qiao', Wei Liu, Changyong Wang and Yuwei Zhao* \\ Tissue Engineering Research Center, Academy of Military Medical Sciences and Department of Neural Engineering and \\ Biological Interdisciplinary Studies, Institute of Military Cognition and Brain Sciences, Academy of Military Medical Sciences, \\ Beijing, China
}

OPEN ACCESS

Edited by:

Miao He,

Fudan University, China

Reviewed by:

Bo Peng

Chinese Academy of Sciences (CAS),

China

Jennie Leach

University of Maryland, Baltimore

County, United States

*Correspondence:

Yuwei Zhao

zhaoyuwei158@163.com

${ }^{\dagger}$ These authors have contributed

equally to this work

Specialty section:

This article was submitted to

Neural Technology,

a section of the journal

Frontiers in Neuroscience

Received: 23 July 2020

Accepted: 15 October 2020

Published: 19 November 2020

Citation:

Zhu H, Qiao X, Liu W, Wang C and Zhao Y (2020) Microglia Play an

Essential Role in Synapse Development and Neuron Maturation in Tissue-Engineered Neural Tissues.

Front. Neurosci. 14:586452.

doi: 10.3389/fnins.2020.586452
In the process of constructing engineered neural tissues, we often use mixed primary neural cells, which contain microglia in the cell culture. However, the role that microglia play in the construction of engineered neural tissue has not been well studied. Here, we generated three-dimensional (3D) engineered neural tissues by silk fibroin/collagen composite scaffolds and primary mixed cortical cells. We depleted microglial cells by magnetic separation. Then, we analyzed the neural growth, development, mature and synapse-related gene, and protein expressions compared with the control engineered neural tissues with the microglia-depleted engineered neural tissues. We found that the engineered neural tissues constructed by magnetic separation to remove microglia showed a decrease in the number of synaptic proteins and mature neurons. These findings link microglia to neuron and synaptic maturation and suggest the importance of microglia in constructing engineered neural tissues in vitro.

Keywords: 3D culture, microglia, neuron, reconstruction, tissue engineering, silk fibroin

\section{INTRODUCTION}

Most of the in vitro approaches for constructing neuronal networks are based on twodimensional (2D) cultures, which cannot recapitulate three-dimensional (3D) organizations, cellcell interaction, or their network functions in vivo (Swistowski et al., 2010; Yang et al., 2016; Martin et al., 2018). In vitro methods to construct 3D neuronal networks that mimic both the structures and functions of neural tissues have been pursued by researchers in various fields, such as neural tissue engineering, neurodegenerative disease studies, and artificial intelligence. Several 3D in vitro research models in the form of cerebral organoids (Lancaster et al., 2013; Bagley et al., 2017; Birey et al., 2017), neurospheroids (Fennema et al., 2013; Jeong et al., 2015; van Pel et al., 2018), and hydrogel cultures (Irons et al., 2008; Ma et al., 2008; Koutsopoulos and Zhang, 2013; Sun et al., 2017) have been developed to study the specific processes of the brain that are challenging to investigate and manipulate in vivo in animal models or ex vivo in brain slices.

Silk protein has received recent attention for neural tissue engineering applications due to its excellent biocompatibility, controllable degradability, controllable mechanical properties, and the ability to be processed into multiple material forms (Kasoju and Bora, 2012; Kundu et al., 2013; Abbott et al., 2016; Huang et al., 2018). The unique silk protein properties protect the neurons from excitotoxicity and maintenance of adequate transfer because of biocompatibility and porous 
structure. The construction of the bioengineered neural tissues based on the silk fibroin (SF)-collagen composite scaffold can recapitulate functional neural networks (Tang-Schomer et al., 2014; Chwalek et al., 2015a,b).

The significant categories of cell sources for 3D neural cultures are pluripotent stem cells, neural stem cells, or primary cortical cells. Researchers often do not use a single type of cell for cell seeding, mainly because brain tissue is composed of different types of cells. In vitro, different types of brain cells play distinct roles in the process of neural cell remodeling and reassembly. Microglia are glial cells from the myeloid lineage. When brain tissue is damaged, microglia migrate to the site of damage and engulf the cellular debris. The recent discovery is that microglia also play some role in the uninjured brain. Microglia have shown that they can remove dead neurons as well as synapses (Schafer et al., 2012). New evidence suggests that microglia in the brain play an essential role in the synaptic organization, control of neuronal excitability, and trophic support during brain development (Stephan et al., 2012; Parkhurst et al., 2013; Grabert et al., 2016; Weinhard et al., 2018). However, in the tissueengineered neural tissue model, the function of microglia, the neuron-microglia interactions are still unclear.

Here, we aimed to study the influence of microglia removal on neuronal survival, synapse development, and functional maturation of the neural network in engineered neural tissue constructed in vitro and tried to explain the interaction relationship between microglia and neurons in engineered neural tissue.

\section{MATERIALS AND METHODS}

\section{Preparation and Characterization of Silk Fibroin Porous Scaffolds}

The silk solution was prepared as previously described (Rockwood et al., 2011). Briefly, silkworm cocoons were boiled in $0.02 \mathrm{M} \mathrm{Na}_{2} \mathrm{CO}_{3}$ for $30 \mathrm{~min}$ and washed with $\mathrm{ddH}_{2} \mathrm{O}$ to extract the sericin. The degummed silk was dissolved in 9.3 $\mathrm{M}$ Libr at $60^{\circ} \mathrm{C}$ for $4-6 \mathrm{~h}$. The solution was dialyzed in $\mathrm{ddH}_{2} \mathrm{O}$ for $48 \mathrm{~h}$ and centrifuged at 12,000 rpm for $30 \mathrm{~min}$ to remove the aggregates. Then, $4 \mathrm{~g} \mathrm{NaCl}$ (particle size $\sim 500 \mu \mathrm{m}$ ) was added to $2 \mathrm{ml}$ of $8 \%$ SF solution in the containers for $48 \mathrm{~h}$. The mixture was immersed in water for $48 \mathrm{~h}$ to extract the $\mathrm{NaCl}$. Porous scaffolds were made as previously described (Altman et al., 2003). The SF scaffolds were sterilized and coated with poly-L-lysine before cell seeding.

The mechanical properties of the SF scaffolds were tested by an Instron mechanical tester (Instron 5900) and compared with rat and mouse brain tissues. All samples were applied $0.2 \mathrm{~N}$ load and compressed by the stress-relaxation tests, which was compressed stepwise at $5 \%$ of the height and relaxed for $500 \mathrm{~s}$ to establish equilibrium. The compressive load-compressive strain diagram was recorded. The compressive modulus was calculated as the minimum linear slope. All scaffolds and tissue samples were cut into $5 \mathrm{~mm}$ diameter and $2 \mathrm{~mm}$ height. Adult rat and mouse brain tissues were dissected from adult animals, stored in sterile PBS at $4^{\circ} \mathrm{C}$, and tested within $4 \mathrm{~h}$ of animal euthanasia.
The SF scaffolds were dehydration treated, and then the morphology was analyzed by scanning electron microscopy (Hitachi S-3400N) at $5 \mathrm{kV}$. Samples were placed onto a copper plate, and gold sputtering was treated on the samples before observation.

\section{Isolation of Primary Cortical Cells}

Isolation of primary cortical cells was assessed as previously described (Pacifici and Peruzzi, 2012). Briefly, primary rat cortical cells were isolated from embryonic day 16-18 SD rats. Cortical tissues were isolated, dissociated with trypsin (0.05\%) followed with trypsin inhibition, centrifuged, and resuspended in NeuroBasal media with B-27 supplement and $2 \mathrm{mM}$ L-glutamine.

\section{Magnetic Labeling and Separation of Microglial Cells}

The isolated primary cortical cells were centrifuged and suspended in $80 \mu \mathrm{l}$ buffer [0.5\% bovine serum albumin (BSA) in phosphate buffered saline (PBS)] and $20 \mu \mathrm{l} \mathrm{CD11b/c} \mathrm{Microbeads}$ (MACS, rat, 130-105-634) per $1 \times 10^{7}$ cells for $15 \mathrm{~min}$ at $4^{\circ} \mathrm{C}$ and then resuspended in $500 \mu \mathrm{l}$ buffer. LD column was placed in the magnetic field of a MACS Separator. The cell suspension was applied onto the column, and the flow-through unlabeled cells were collected, which were called microglia-depleted cortical cells. The magnetically labeled cells flushed out were microglia. The microglia were grown in Dulbecco's Modified Eagle's Medium (DMEM)-F12 plus 10\% fetal bovine serum (FBS).

\section{Construct Assembly, Culture, and Evaluation}

The concentrated cortical cells and microglia-depleted cortical cells were seeded on the SF scaffolds (5 $\mathrm{mm}$ diameter, $1 \mathrm{~mm}$ height) for $24 \mathrm{~h}$, immersed with $200 \mu \mathrm{l}$ collagen $(3 \mathrm{mg} / \mathrm{ml})$ for $30 \mathrm{~min}$ at $37^{\circ} \mathrm{C}$, and adjusted $\mathrm{pH}$ to 7 by $\mathrm{NaOH}$. The inside and edges of the scaffolds were immersed in collagen, and there were no bubbles in the added collagen. The construct seeded mixed cortical cells were called the control group, and the construct seeded microglia-depleted cortical cells were called the depleted group. For control cultures used for cell viability measurement and axon length assays, cells were seeded at $1 \times 10^{6}, 5 \times 10^{6}$, and $1 \times 10^{7}$ cells/composite scaffold. For control cultures and microglia-depleted group used for microtubule-associated protein 2 (MAP2) positive immunofluorescence staining, neural maturation, synapse development-related gene, and protein expressions based on SF/collagen scaffolds, cells were seeded at $5 \times 10^{6}$ cells/composite scaffold.

\section{Cell Viability Evaluation}

A Live/Dead Kit (Invitrogen) was used to evaluate cell viability. Constructs were incubated with Calcein AM and EthD- 1 at $37^{\circ} \mathrm{C}$ for 15-30 $\mathrm{min}$. After incubation, cells were washed three times with PBS and imaged through a confocal microscope (Nikon A1). The images were taken from the surface to $10 \mu \mathrm{m}$ deep at $2 \mu \mathrm{m}$ intervals.

Cell Counting Kit-8 (CCK-8) assay (Dojindo) was used to assess the cell viability of $3 \mathrm{D}$ engineered tissue cultures and $2 \mathrm{D}$ 
cultures. CCK-8 was mixed in culture media (1:10) at different time points (day in vitro 1,3 , and 7 and one time every week up to 6 weeks) and incubated for $2 \mathrm{~h}$ at $37^{\circ} \mathrm{C}$. Fluorescence was read at $450 \mathrm{~nm}$ on a microplate spectrophotometer (Molecular Devices). Three samples were used for each experiment.

\section{Immunofluorescence Staining}

After harvest, the samples were fixed with 4\% paraformaldehyde for $30 \mathrm{~min}$, washed with PBS, and treated with $0.3 \%$ Triton X100 including 5\% normal goat serum for $30 \mathrm{~min}$, followed by incubation of primary antibodies overnight at $4^{\circ} \mathrm{C}$. The next day after three 5 min washes, samples were incubated with secondary antibodies for $2 \mathrm{~h}$ at room temperature. Antibodies included anti-Tuj1, anti-CD11b/c, anti-IBA1, anti-MAP2, and goat antimouse or anti-rabbit secondary antibodies. Table 2 shows the information of the primary antibodies. Cell nuclei were stained with DAPI. Samples were again washed thoroughly with PBS before imaging. Fluorescence images were acquired by a Nikon A1 confocal microscope. The images were taken from the surface to 50-60 $\mu \mathrm{m}$ deep at $5 \mu \mathrm{m}$ intervals.

\section{Real-Time PCR Analysis}

We assessed the expression level of genes (NCAM-L1, MAP2, Tuj1, NF-L, synaptophysin, GABAR1, etc.) associated with the neuron growth and microglial inflammatory response using realtime PCR. Briefly, total RNA was extracted with TRIzol reagent, and PCRs were carried out using a LightCycler 96 real-time quantitative thermal cycler (Roche) with SYBR Green PCR Mix (Toyobo). GAPDH was used as an internal reference. Table 1 shows all the primer sequences used in this study. All reactions were run in triplicate. The fluorescence intensity was recorded under the setting as follows: $20 \mathrm{~s}$ at $95^{\circ} \mathrm{C}$ and $30 \mathrm{~s}$ at $58^{\circ} \mathrm{C}$ for 40 cycles. Finally, the gene expression value of individual constructs was carefully calculated relative to $G A P D H$ expression using the $2^{-\Delta \Delta C t}$ method.

\section{Western Blotting}

Western blot analysis was carried out using control or microgliadepleted samples from day 7 and day 14 engineered tissues. The samples were treated in liquid nitrogen for $20 \mathrm{~min}$, and then proteins extracted by Laemmli were homogenized on ice.
TABLE 2 | Information of antibodies used in this study.

\begin{tabular}{lccc}
\hline Antibody & Type & Dilution & Source \\
\hline Tuj1 & Poly-rabbit & $1: 500$ & Abcam ab18207 \\
CD11b/c & Poly-mouse & $1: 200$ & Abcam ab1211 \\
MAP2 & Poly-rabbit & $1: 500$ & Abcam ab32454 \\
IBA1 & Poly-mouse & $1: 200$ & Abcam ab15690 \\
Synaptophysin & Poly-rabbit & $1: 200$ & Abcam ab32127 \\
\hline
\end{tabular}

TABLE 3 | Abbreviations that appeared in this study.

\begin{tabular}{ll}
\hline Acronyms & Full acronym \\
\hline NCAM & Neural cell adhesion molecule \\
GAP-43 & Growth-associated protein-43 \\
SNP-25 & Synaptosomal-associated protein-25 \\
SF & Silk fibroin \\
3D & Three-dimensional \\
2D & Two-dimensional \\
CNS & Central nervous system \\
MAP2 & Microtubule-associated protein 2 \\
BSA & Bovine serum albumin \\
GABA & Gamma aminobutyric acid \\
\hline
\end{tabular}

The supernatant was collected after centrifugation at $12,000 \mathrm{rpm}$ for $10 \mathrm{~min}$. The concentration of proteins was detected by BCA Protein Assay Kit (Invitrogen). Equal amounts (60 $\mu \mathrm{g})$ were separated on $12 \%$ SDS-PAGE gels and transferred to nitrocellulose membrane. The membranes were blocked with $5 \%$ defatted milk for $1 \mathrm{~h}$, incubated with primary antibodies overnight at $4^{\circ} \mathrm{C}$, and incubated with appropriate secondary antibodies for $1 \mathrm{~h}$ at room temperature. Table 2 shows the information of the primary antibodies. The labeled proteins were detected by enhanced chemiluminescence reagent (Applygen). GAPDH was used as the control to correct the band intensity.

\section{Statistical Analyses}

All quantitative analyses were performed at least in triplicate, and the mean values were obtained. Results presented were based on the averages of data and standard error of the mean as error bars. The analysis used the Student's $t$-test.

TABLE 1 | Primer sequences in this study.

\begin{tabular}{|c|c|c|}
\hline Genes & Forward primer & Reverse primer \\
\hline Tuj1 & GCCAAGTTCTGGGAGGCTCATC & GTAGTAGACACTGTAGCGTTCCA \\
\hline GAP-43 & AACGGAGACTGCAGAAAGCA & GCCTCGGGGTCTTCTIACC \\
\hline SNP-25 & TGGATGAGCAAGGCGAACAA & TCCTGATTATTGCCCCAGGC \\
\hline NCAM & CACCAGTGAGAGGGTGAGTG & CTCCAGTACATGGTGTCCTाT \\
\hline NF-L & AATAAGTCGACGCTGCAGGACCTCAACCA & GATCTGAATTCCTGAGCCTGGTCTCTTC \\
\hline MAP2 & GAGAAGGAGGCCCAACACAA & TCTTCGAGGCTTCTTCCAGTG \\
\hline IL-10 & TाTAGGCGAGAAGCTGAAGG & TCTTCACAGGGCAGGAATCT \\
\hline $\mathrm{IL}-1 \beta$ & GACCTGTTCTIGAGGCTGAC & TAGCCACGCCTTCTGTGACTCTAACT \\
\hline TGF- $\beta 1$ & CCCGCATCCCAGGACCTCTCT & CGGGGGACTGGCGAGCCTTAG \\
\hline IL-6 & GACTGATGTTGTTGAGAGCCACTG & TAGCCACGCCTTCTGTGACTCTAACT \\
\hline Synaptophysin & CATTCATGCGCGCACCTCCA & TTGCTGCCCATAGTCGCCCT \\
\hline GABAR1 & GTGCAAGTTAAATTGCGCTGCA & GCTTCCCAATATCCAATCTGCAGC \\
\hline GAPDH & GATGGTGAAGGTCGGTGTGA & GGGATCTCGCTCCTGGAAG \\
\hline
\end{tabular}




\section{Ethics Statement}

All animal care and experimental protocols complied with the Animal Management Rule of the Ministry of Health, People's Republic of China (Documentation No. 55, 2001). All procedures were approved by the Institutional Animal Care and Use Committee of the Academy of Military Medical Sciences, Beijing, China.

Table 3 shows all the abbreviations that appeared in this study.

\section{RESULTS}

\section{Scaffold Characterization}

The overall fabrication process from SF extraction to aqueousbased sponges is shown in Figure 1A. The pore size of the scaffolds was about $<500 \mu \mathrm{m}$. The bright-field microscope image (Figure 1B) and SEM image (Figure 1C) showed the high porosity of the scaffolds, which allowed sufficient exchange of nutrients, oxygen, and wastes. The mechanical stiffness of the scaffolds and the cortical tissues of mouse and rat were tested by confined compression tests. The respective load-strain trace is shown in Figure 1D. The SF scaffolds had a modulus of $32.2 \pm 9.7 \mathrm{kPa}$ higher than a rat brain of $12.1 \pm 2 \mathrm{kPa}$ and a mouse brain of $3.1 \pm 0.1 \mathrm{kPa}$.

\section{Biofabrication of 3D Engineered Neural Tissues}

Since we have fabricated the SF sponges, the isolated primary cortical cells were cultured on the SF sponge. After the cortical cells were attached to the SF sponges, the scaffolds were filled with the collagen matrix to allow axon outgrowth and network formation. The 3D constructs viability of different cell seeding densities at 3 days of culture were stained with Live/Dead to evaluate the cell viability (Figures $2 \mathrm{~A}, \mathbf{B}$ ). Confocal images showed that the dead cells percentage gradually increased as the cell seeding density increased. The 3D constructs growth of different cell seeding densities after 7 days of culture were immunostained with anti-Tuj1 fluorescent. The results showed that the protein expression of Tuj1 protein of different cell seeding densities was dissimilar. As cell seeding density increases, the more complex the neural network is formed morphologically (Figures 2C,D). The engineered neural tissue viability and axon outgrowth were found to be affected by cell density. Figure 2 shows that the most suitable cell seeding density was $5 \times 10^{6}$ cells/scaffold, which showed the prolonged axon and higher proportion of live cells.

\section{D Engineered Neural Tissues Growth}

Further, we observed the cells viability, axon growth of the engineered neural tissues in vitro compared with $2 \mathrm{D}$ culture. By confocal images, we observed that the axon growth of the

A

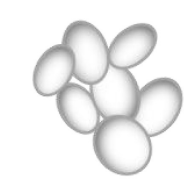

Silkworm cocoons

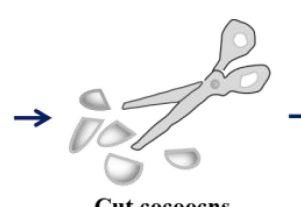

Cut cocoocns

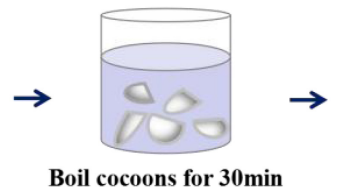

Boil cocoons for $30 \mathrm{mi}$ in $0.02 \mathrm{M} \mathrm{Na}_{2} \mathrm{CO}_{3}$

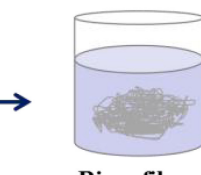

Rinse fibers for 3 times

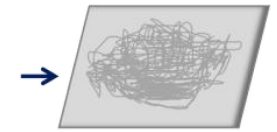

Dry thoroughly

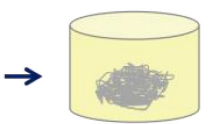

Dissolve fibers in 9.3M LiBr at $60^{\circ} \mathrm{C}$
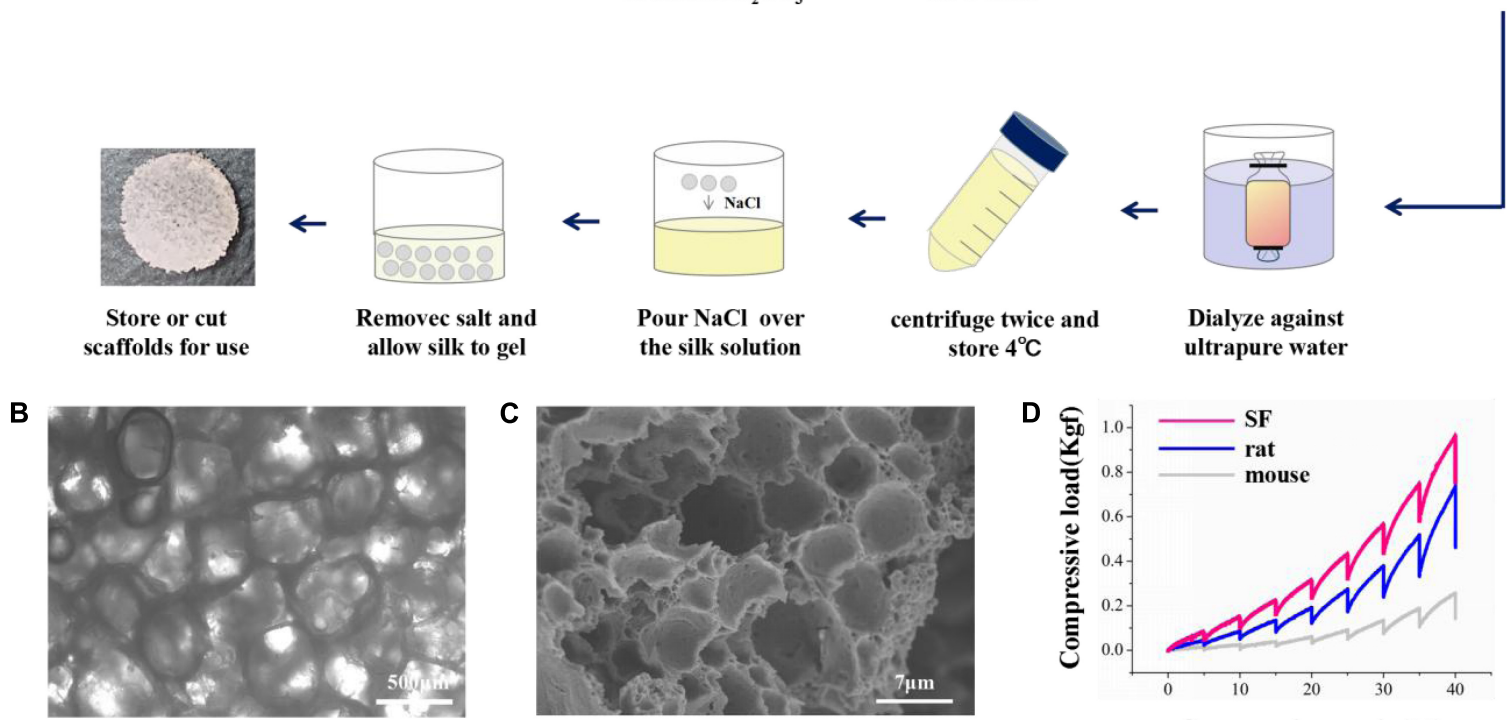

Dialyze against ultrapure water store $4^{\circ} \mathrm{C}$

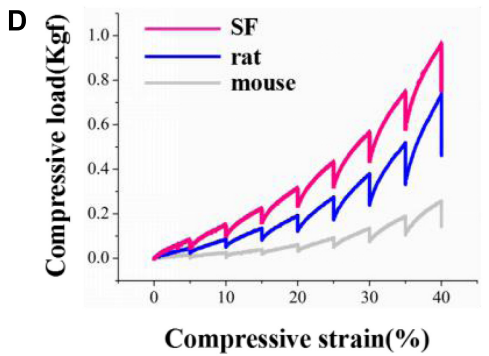

FIGURE 1 | The preparation and characterization of the silk fibroin porous scaffolds. (A) Schematic from the silk fibroin extraction to the porous scaffolds. It takes about 4 days from the silk cocoons to the final silk fibroin porous sponges. (B,C) The bright-field microscope image and the scanning electron microscopy image show the inner structure of the SF sponges. (D) Compressive load-strain traces of the SF sponge, rat, and mouse cortical tissues. 


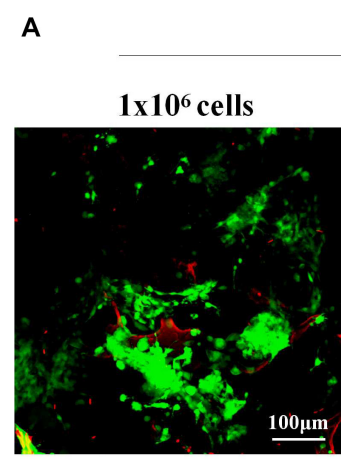

C

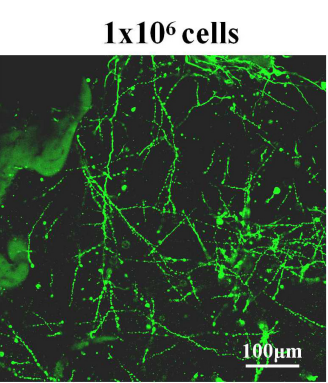

Day 3: Live/Dead Staining

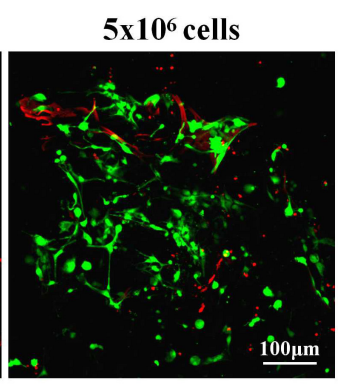

Day7: Axon outgrowth

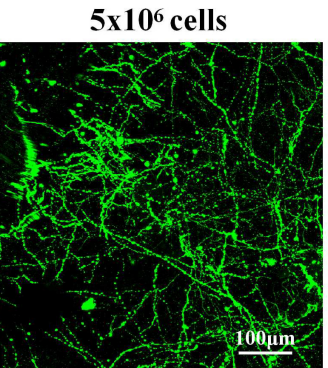

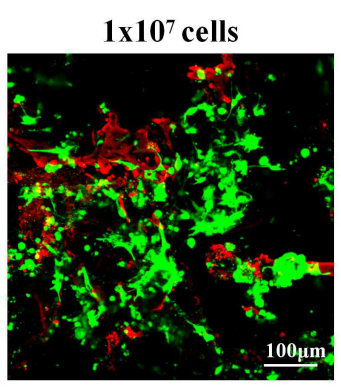

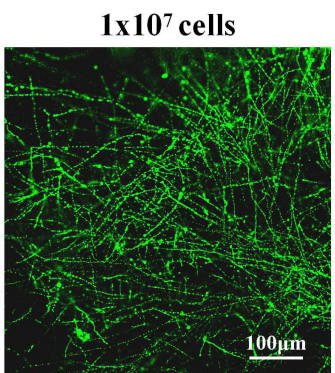

B

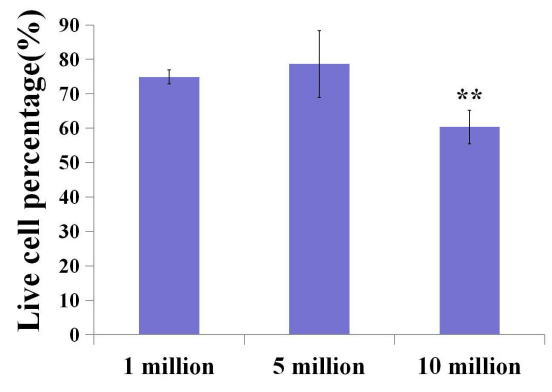

Cell number per scaffold

D

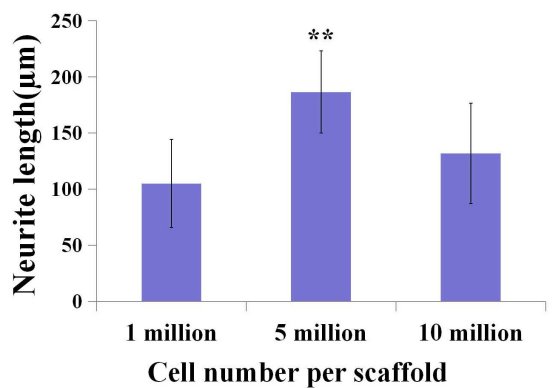

FIGURE 2 | The live cell percentage and axon length with different cell densities. (A,B) Live (green)/Dead (red) staining showed live cell percentage with different cell densities on the SF sponges on day 3. (C,D) Immunochemistry with anti-Tuj1 indicated the neuronal axon length of different cell densities at day $7 .{ }^{\star \star} p<0.01$.

A
2D - day 7
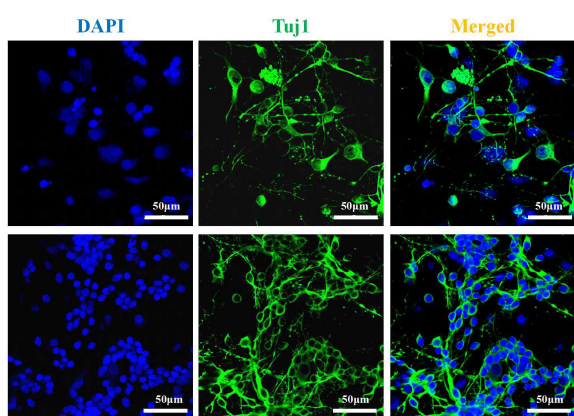

B

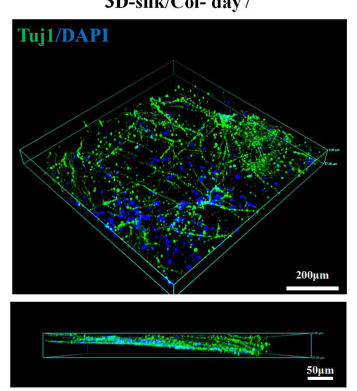

3D-silk/Col- day14

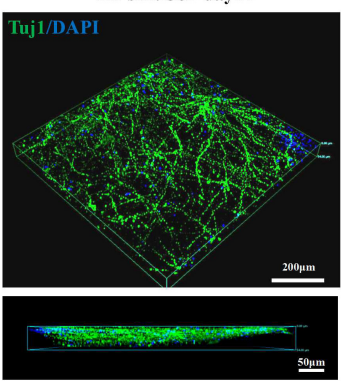

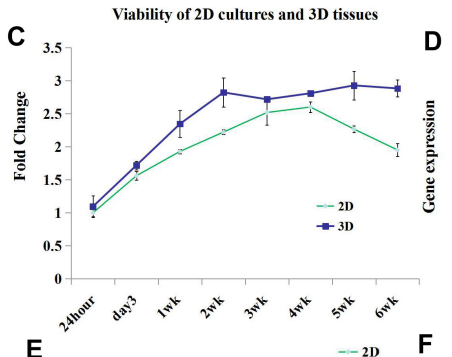

E

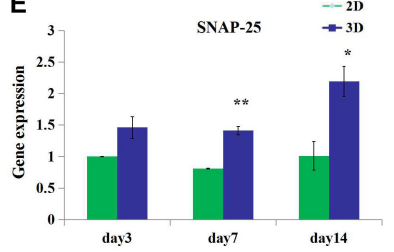

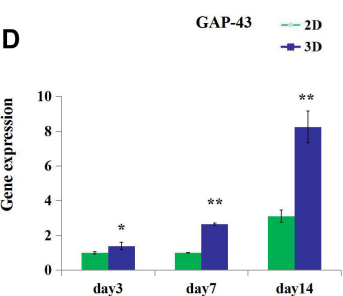

$\mathbf{F}$

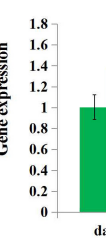

NCAM $\quad-2$ -

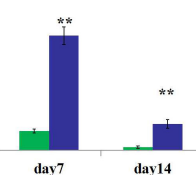

FIGURE 3 | 3D engineered neural tissue growth compared with 2D cultures. (A) Fluorescence images of 2D cultures on day 7 and day 14. (B) Fluorescence images of 3D fluorescence images of confocal z stacks of day 7 and day 14 neuronal axon on the SF sponges immunostained with Tuj1 in green and DAPI in blue. (C) Viability of 2D cultures and 3D engineered neural tissue cultures at 24 h, 3, 7 days, and 2-6 weeks assayed with CCK-8 and expressed relative to 24-h levels. (D-F) Expression of growth-associated protein 43 (GAP-43), neural cell adhesion molecule L1 (NCAM-L1), and synaptosomal-associated protein 25 (SNP-25) mRNA in 2D culture (green) and 3D engineered neural tissues culture (deep blue) at day 7 and day 14 relative to day 3 expression. ${ }^{*} p<0.05$ and ${ }^{* \star} p<0.01$. 
engineered neural tissues was opposite from the $2 \mathrm{D}$ cultures (Figures 3A,B). The nucleus was anchored on the surface of porous sponges, and the axons penetrated the gel along with culture time. $3 \mathrm{D}$ engineered neural tissue culture showed a more prolonged axon than $2 \mathrm{D}$ culture.

The viability of $3 \mathrm{D}$ engineered neural tissues and $2 \mathrm{D}$ cultures was assayed by the CCK- 8 at 24 h, 3, 7 days, 2, 3, 4, 5, and 6 weeks. $3 \mathrm{D}$ engineered neural tissue culture showed higher viability by OD value (Figure 3C). After 4 weeks, cortical cells viability in $2 \mathrm{D}$ cultures showed a decrease, and cortical cells in $3 \mathrm{D}$ cultures still maintained high viability. This result demonstrated that the SF scaffold would promote long-term cell survival compared with $2 \mathrm{D}$ cultures. Gene expression also showed the differences between $2 \mathrm{D}$ cultures and 3D cultures.

After 3 days of culture, the expression of growth-associated protein 43 (GAP-43) and NCAM-L1 was observed to be significantly upregulated for $3 \mathrm{D}$ cultures grown on $\mathrm{SF} /$ collagen composite materials compared with 2D cultures (1.4-fold). Similarly, after 7 days of culture, the expression of GAP43, SNAP-25, and NCAM-L1 was observed to be significantly upregulated for $3 \mathrm{D}$ cultures compared with 2D cultures (2. 7-, 1. 8-, and 6-fold, respectively). The calculated expression levels of GAP-43, SNAP-25, and NCAM-L1 at 14 days of culture amounted to 2. 7-, 2. 2-, and 8.8-fold for 3D cultures compared with 2D cultures (Figures 3D-F). 3D engineered neural tissues showed higher expression levels of regenerative growth (GAP-43), synaptogenesis (SNAP-25), and neuronal adhesion (NCAM-L1) compared with 2D cultures at day 3, day 7 , and day 14 .

\section{Depletion of Primary Microglia by Magnetic Separation}

Schematic images of magnetic separation of primary microglia are shown in Figure $\mathbf{4 A}$. Immunofluorescence studies were performed to evaluate the depletion of the microglia after magnetic separation. The magnetically labeled cells flushed out showed IBA1 positive, indicating that the labeled cells were microglia, and most of the microglia were depleted by magnetic separation. We called the engineered neural tissues containing microglia the control group and the engineered neural tissues depleting microglia the depleted group (Figure 4B).

\section{The Maturation of Neurons and Synapse Development Were Decreased in the Microglia-Depleted 3D Tissues}

By flow cytometry (Figure 5A), the proportion of the neurons in the isolated primary cortical cells is about

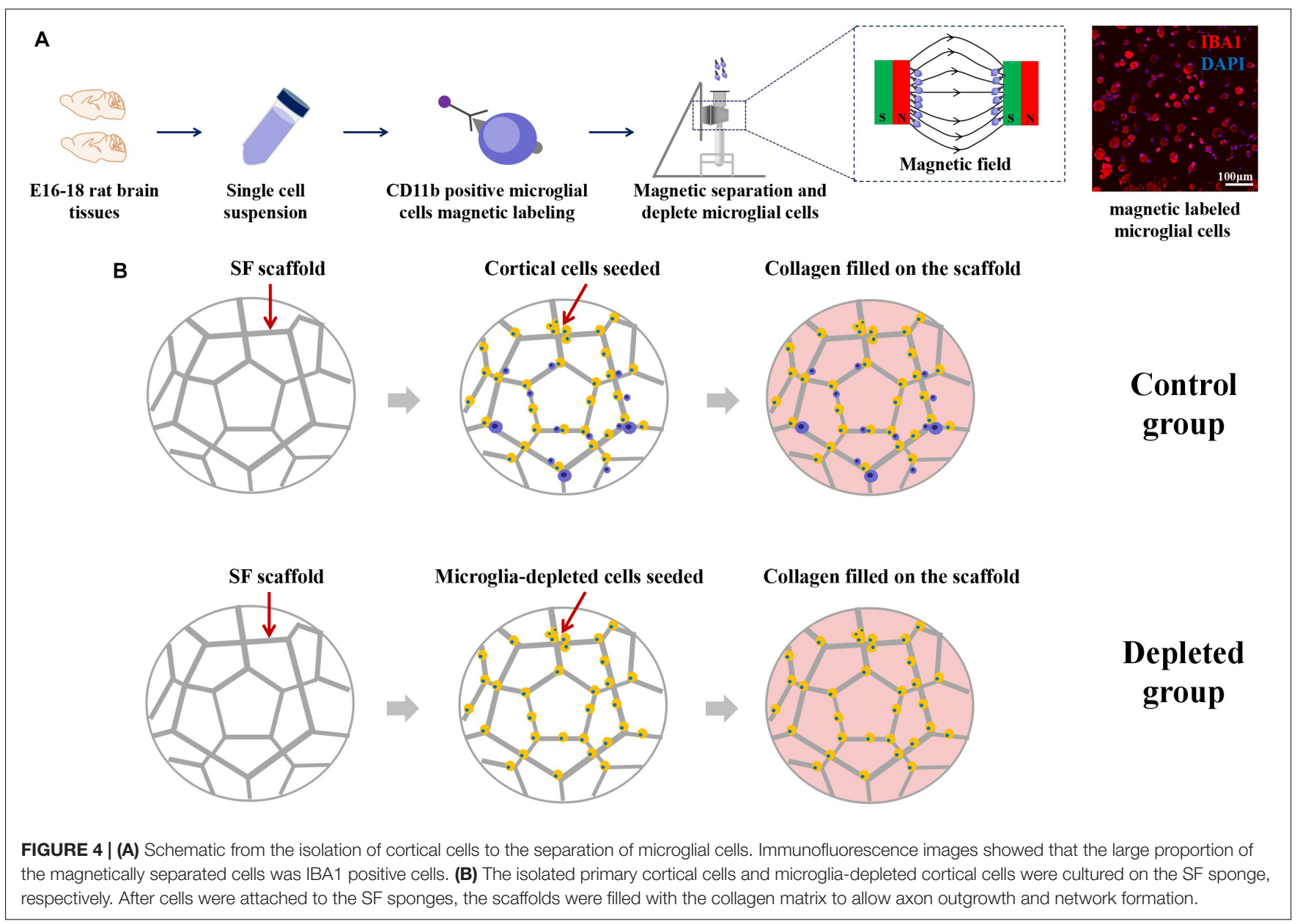



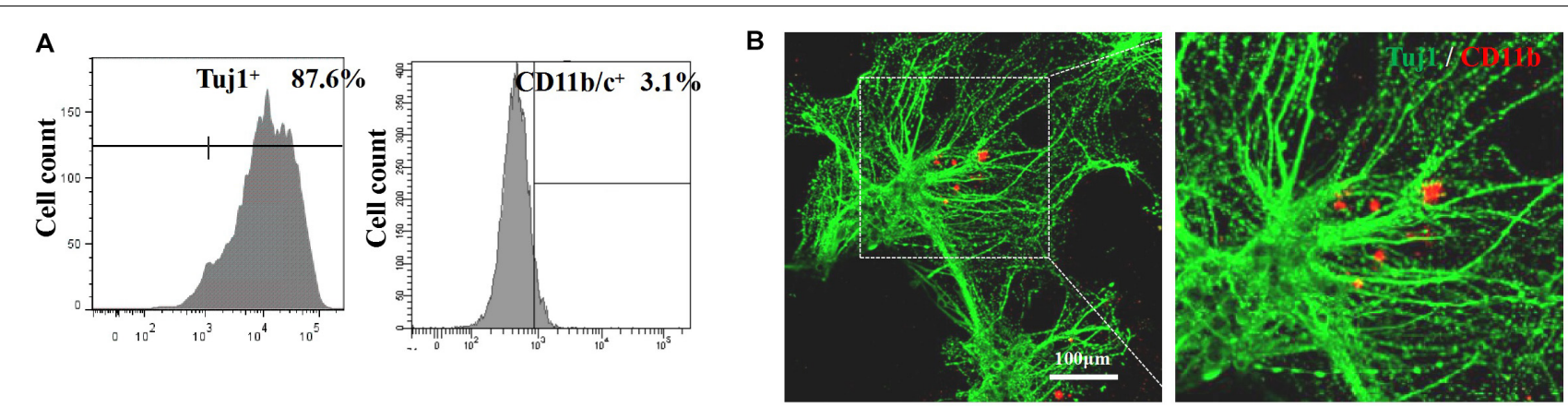

c

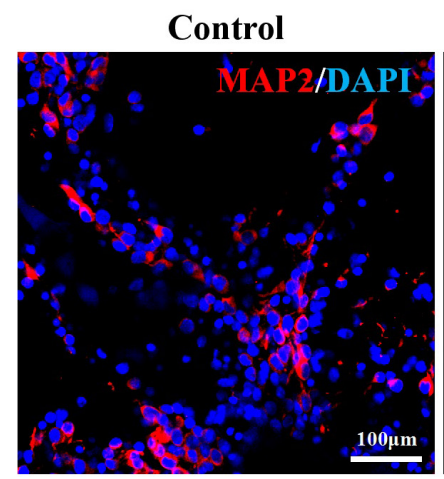

Microglia-depleted

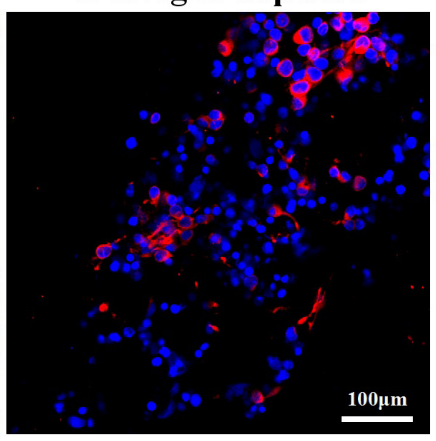

D

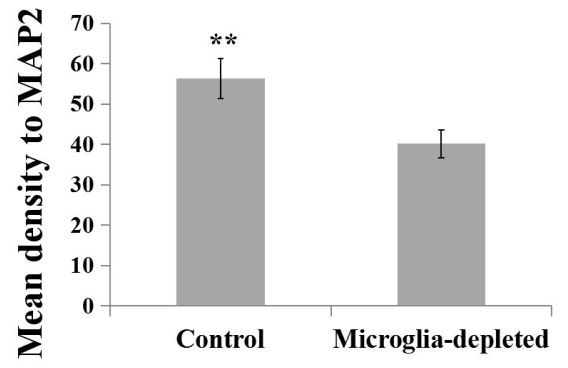

FIGURE 5 | (A,B) Proportion and morphological distribution of neurons and microglia of the primary cortical cells. (C) Fluorescence images of day 7 axons immunostained with MAP2 in red and DAPI in blue. (D) Expression of microglia-related pro-inflammatory factor (IL-1 $\beta$, IL-6) and inflammatory factor (IL-10, TGF- $\beta$ ) in the 3D engineered neural tissues (control group) and 3D engineered microglia-depleted neural tissues (depleted group). ${ }^{* *} p<0.01$.

$87.6 \pm 5.2 \%(n=3)$; the proportion of microglia is about $3.1 \pm 0.25 \%(n=3)$. To visualize microglia and neuron in the engineered neural tissues, we labeled microglia with $\mathrm{CD} 11 \mathrm{~b} / \mathrm{c}$, a neuron with Tuj1 using immunofluorescence staining. The Tuj1 and CD11b/c double-stained results of the control group revealed that in some cases, microglia were surrounded by neuron cell body and axon (Figure 5B). After 7 days of culture, the expression levels of MAP2 of the depleted group were lower than those of the control group (Figures 5C,D).

$3 \mathrm{D}$ neural tissues (control group) tended to have higher expression level of the neuron (Tuj1), mature neuron (MAP2), neuronal adhesion molecular (NCAM-L1), synaptogenesis (synaptophysin), neurofilament (NF-L), and receptors for gamma-aminobutyric acid (GABAR1) than the microgliadepleted 3D neural tissues (depleted group) as shown in Figure 6A. Especially after 4 days of culture, the expression of GABAR1 and Tuj1 was significantly upregulated for the control group compared with the microglia-depleted group (2.9- and 2.5-fold, respectively). The expression of NF-L after 7 days and GABAR1 after 14 days was significantly upregulated for the control group compared with the microglia-depleted group (4.3- and 2.4-fold, respectively). The expression levels of MAP2 and synaptophysin of the depleted group were lower than those of the control group at day 7 and day 14, respectively. These results of gene expression were consistent with the results of protein expression by Western blot (Figure 6B), suggesting that microglia play an essential role in synapse development and neuron maturation in tissue-engineered neural tissues.

In summary, we generated 3D engineered neural tissues based on the SF-collagen scaffolds. We compared the viability, the axon outgrowth length, and the expression from different cell densities. On this basis, we depleted microglia by magnetic separation and seeded the cortical cells, which remove microglia on the SF-collagen composite scaffold. The effect of the microglia depletion on the structure, gene, and protein expression was determined by immunocytochemical method, mRNA gene expression, and Western blot. We demonstrated that the maturation of neurons and synapse development were decreased in the microglia-depleted 3D tissues group compared with the control group. It is proven that microglia play an essential role in synapse development and neuron maturation in tissueengineered neural tissues.

\section{DISCUSSION}

In this study, we mainly studied the role of microglia in the construction of 3D tissue-engineered neural tissues. We found that the depletion of microglia affects the synapse development and neuron maturation in the $3 \mathrm{D}$ neural tissues. This indicated that it is best to use mixed cortical cells of cells differentiated from neural progenitor/stem cells as seeded cells in the construction of engineered neural tissues, which is beneficial to constructing the functional engineered organization. 


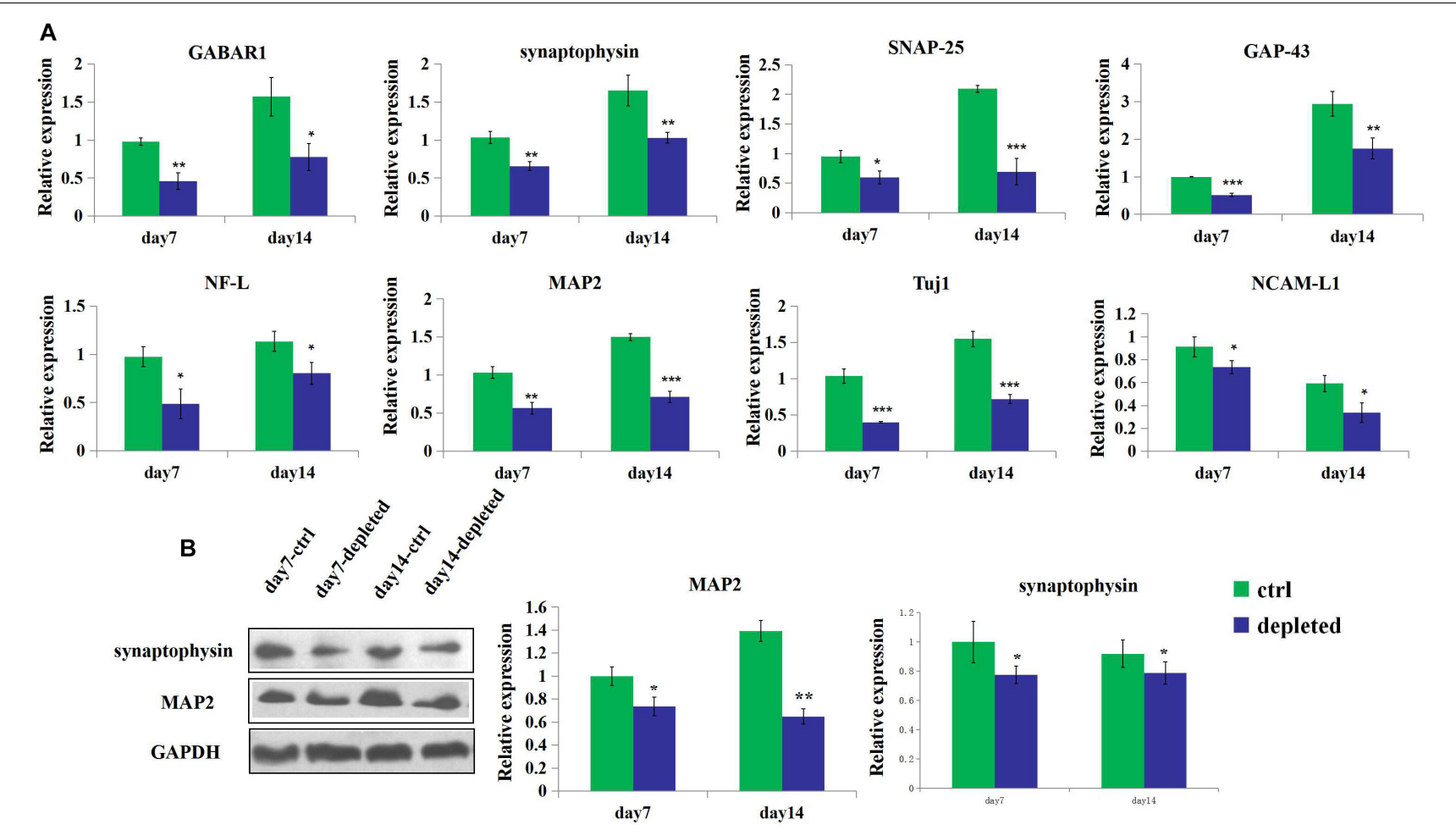

FIGURE 6 | Gene and protein expression of the 3D engineered neural tissues. (A). Expression of GABAR1, synaptophysin, SNAP-25, GAP-43, NCAM-L1, NF-L, MAP2, and Tuj1 mRNA in the control group (light blue) and the microglia-depleted group (deep blue) at day 14 relative to the day 7 expression. (B) Expression of synaptophysin, MAP2, and GAPDH protein in the control group (green) and the microglia-depleted group (deep blue) at day 14 relative to the day 7 expression. ${ }^{\star} p<0.05,{ }^{* \star} p<0.01$, and ${ }^{* * *} p<0.001$.

Microglia are homologous macrophage cells that, in addition to providing surveillance and clearance role, can engulf synapses in uninjured brains and achieve synaptic pruning during postnatal development (Prinz and Priller, 2014; Dong et al., 2018; Jung and Chung, 2018; Surinkaew et al., 2018). Paolicelli et al. demonstrated that microglia are involved in the development of brain wiring in newborn mice, and that disrupting microgliasynapse interactions delays the maturation of synaptic circuits (Paolicelli et al., 2011; Norris et al., 2018).

Engineered neural tissue construction is a hot topic in recent years. In recent years, researchers studied microglia-related inflammation or fractal analysis in tissue engineering (Jiang et al., 2016; Koss et al., 2017). However, until now, the role of microglia in the engineered neural tissue construction in vitro has not been clarified. Clarifying the role of microglia in the $3 \mathrm{D}$ engineered neural tissues contributes significantly to the engineered tissue construction and 3D models in neuroscience.

Obtaining or isolating microglia in vitro is different because of the cellular heterogeneity. Researchers usually use the method that is divided into three sections: mixed microglial, astroglial, and oligodendroglial cell culture; culture maintaining; and isolation of other cells except for microglia by shaking or digestion method (Ni and Aschner, 2010; Tamashiro et al., 2012; Roy, 2018). Here, we used a magnetic separation for isolation of primary microglia from primary mixed cortical cells. This method can highly and efficiently remove pure microglia from the mixed cortical cells by magnetic separation. The removed microglia obtained by this method were fully functional and morphologically like microglia obtained by conventional isolation methods.

In summary, microglia play a critical role in synapse development and neuron maturation in tissue-engineered neural tissues. Based on our findings, the maturation of neurons and synapse development were decreased in the microglia-depleted 3D bioengineered neural tissues. Microglia affect engineered neural tissues neuron maturation and synapse development probably through three ways: (1) microglia can remove dead and dying neurons and further affect the viability and remodeling of the engineered neural tissues; (2) in addition to some proinflammatory and inflammatory factors, microglia also secrete some neurotrophic factors; and (3) microglia remodel, run, or engulf synapse. However, further research is needed to illuminate the mechanisms on how the microglia affect neuron maturation and synapse development and related signal pathway.

\section{DATA AVAILABILITY STATEMENT}

The original contributions presented in this study are included in the article, further inquiries can be directed to the corresponding author. 


\section{ETHICS STATEMENT}

The animal study was reviewed and approved by the Institutional Animal Care and Use Committee of Academy of Military Medical Sciences.

\section{AUTHOR CONTRIBUTIONS}

$\mathrm{HZ}, \mathrm{XQ}$, and $\mathrm{YZ}$ designed the project. $\mathrm{HZ}$ wrote the manuscript and performed the experiments. $\mathrm{HZ}$ and $\mathrm{XQ}$ analyzed the data.

\section{REFERENCES}

Abbott, R. D., Kimmerling, E. P., Cairns, D. M., and Kaplan, D. L. (2016). Silk as a biomaterial to support long-term three-dimensional tissue cultures. ACS Appl. Mater. Interfaces 8, 21861-21868. doi: 10.1021/acsami.5b12114

Altman, G. H. I., Diaz, F., Jakuba, C., Calabro, T., Horan, R. L., Chen, J., et al. (2003). Silk-Based-Biomaterials. Biomaterials 24, 401-416. doi: 10.1016/s01429612(02)00353-8

Bagley, J. A., Reumann, D., Bian, S., Lévi-Strauss, J., and Knoblich, J. A. (2017). Fused cerebral organoids model interactions between brain regions. Nat. Methods 14, 743-751. doi: 10.1038/nmeth.4304

Birey, F., Andersen, J., Makinson, C. D., Islam, S., Wei, W., Huber, N., et al. (2017). Assembly of functionally integrated human forebrain spheroids. Nature 545, 54-59. doi: 10.1038/nature22330

Chwalek, K., Sood, D., Cantley, W. L., White, J. D., Tang-Schomer, M., and Kaplan, D. L. (2015a). Engineered 3D Silk-collagen-based model of polarized neural tissue. J. Vis. Exp. 105:e52970. doi: 10.3791/52970

Chwalek, K., Tang-Schomer, M. D., Omenetto, F. G., and Kaplan, D. L. (2015b). In vitro bioengineered model of cortical brain tissue. Nat. Protoc. 10, 13621373. doi: 10.1038/nprot.2015.091

Dong, P., Zhao, J., Li, N., Lu, L., Li, L., Zhang, X., et al. (2018). Sevoflurane exaggerates cognitive decline in a rat model of chronic intermittent hypoxia by aggravating microglia-mediated neuroinflammation via downregulation of PPAR- $\gamma$ in the hippocampus. Behav. Brain Res. 347, 325-331. doi: 10.1016/j. bbr.2018.03.031

Fennema, E., Rivron, N., Rouwkema, J., van Blitterswijk, C., and de Boer, J. (2013). Spheroid culture as a tool for creating 3D complex tissues. Trends Biotechnol. 31, 108-115. doi: 10.1016/j.tibtech.2012.12.003

Grabert, K., Michoel, T., Karavolos, M. H., Clohisey, S., Baillie, J. K., Stevens, M. P., et al. (2016). Microglial brain region-dependent diversity and selective regional sensitivities to ageing. Nat. Neurosci. 19, 504-516. doi: 10.1038/nn.4222

Huang, W., Ling, S., Li, C., Omenetto, F. G., and Kaplan, D. L. (2018). Silkworm silk-based materials and devices Silkworm silk-based materials and devices generated using bio-nanotechnology. Chem. Soc. Rev. 47, 6486-6504. doi: 10. $1039 /$ c8 $\operatorname{cs} 00187 \mathrm{a}$

Irons, H. R., Cullen, D. K., Shapiro, N. P., Lambert, N. A., Lee, R. H., and Laplaca, M. C. (2008). Three-dimensional neural constructs a novel platform for neurophysiological investigation. J. Neural Eng. 5, 333-341. doi: 10.1088/ 1741-2560/5/3/006

Jeong, G. S., Chang, J. Y., Park, J. S., Lee, S. A., Park, D., Woo, J., et al. (2015). Networked neural spheroid by neuro-bundle mimicking nervous system created by topology effect. Mol. Brain 8:17. doi: 10.1186/s13041-0150109-y

Jiang, Z., Song, Q., Tang, M., Yang, L., Cheng, Y., Zhang, M., et al. (2016). Enhanced migration of neural stem cells by microglia grown on a three-dimensional graphene scaffold. ACS Appl. Mater. Interfaces 8, 25069-25077. doi: 10.1021/ acsami.6b06780

Jung, Y. J., and Chung, W. S. (2018). Phagocytic roles of glial cells in healthy and diseased brains. Biomol. Ther. 26, 350-357. doi: 10.4062/biomolther.2017.133

Kasoju, N., and Bora, U. (2012). Silk fibroin in tissue engineering. Adv. Healthc. Mater. 1, 393-412. doi: 10.1002/adhm.201200097

Koss, K. M., Churchward, M. A., Jeffery, A. F., Mushahwar, V. K., Elias, A. L., and Todd, K. G. (2017). Improved 3D hydrogel cultures of primary glial cells for
CW supervised the project. All authors contributed to the article and approved the submitted version.

\section{FUNDING}

This work was supported by the Key Program of the National Key Research and Development Program of China (no. 2016YFY1101303), the Key Program of National Natural Science Foundation of China (no. 31830030), and the Joint Funds for National Natural Science Foundation of China (no. U1601221). in vitro modelling of neuroinflammation. J. Vis. Exp. 130:56615. doi: 10.3791/ 56615

Koutsopoulos, S., and Zhang, S. (2013). Long-term three-dimensional neural tissue cultures in functionalized self-assembling peptide hydrogels. Matrigel and Collagen I. Acta Biomater. 9, 5162-5169. doi: 10.1016/j.actbio.2012.09.010

Kundu, B., Rajkhowa, R., Kundu, S. C., Wang, X., Kundu, B., Rajkhowa, R., et al. (2013). Silk fibroin biomaterials for tissue regenerations. Adv. Drug Deliv. Rev. 65, 457-470. doi: 10.1016/j.addr.2012.09.043

Lancaster, M. A., Renner, M., Martin, C. A., Wenzel, D., Bicknell, L. S., Hurles, M. E., et al. (2013). Cerebral organoids model human brain development and microcephaly. Nature 501, 373-379. doi: 10.1038/nature12517

Ma, W., Tavakoli, T., Chen, S., Maric, D., Liu, J. L., O’Shaughnessy, T. J., et al. (2008). Reconstruction of Functional Cortical-like Tissues from Neural Stem and Progenitor Cells. Tissue Eng. Part A 14, 1673-1686. doi: 10.1089/ten.tea. 2007.0357

Martin, A. D., Chua, S. W., Au, C. G., Stefen, H., Przybyla, M., Lin, Y., et al. (2018). Peptide nanofiber substrates for long-term culturing of primary neurons. ACS Appl. Mater. Interfaces 10, 25127-25134. doi: 10.1021/acsami.8b07560

$\mathrm{Ni}, \mathrm{M}$., and Aschner, M. (2010). Neonatal rat primary microglia isolation, culturing, and selected applications. Curr. Protoc. Toxicol. 43, 12.17.1-12.17.16. doi: 10.1002/0471140856.tx1217s43

Norris, G. T., Smirnov, I., Filiano, A. J., Shadowen, H. M., Cody, K. R., Thompson, J. A., et al. (2018). Neuronal integrity and complement control synaptic material clearance by microglia after CNS injury. J. Exp. Med. 215, 1789-1801. doi: 10.1084/jem.20172244

Pacifici, M., and Peruzzi, F. (2012). Isolation and culture of rat embryonic neural cells: a quick protocol. J. Vis. Exp. 63:e3965. doi: 10.3791/3965

Paolicelli, R. C., Bolasco, G., Pagani, F., Maggi, L., Scianni, M., Panzanelli, P., et al. (2011). Synaptic pruning by microglia is necessary for normal brain development. Science 333, 1456-1458. doi: 10.1126/science.1202529

Parkhurst, C. N., Yang, G., Ninan, I., Savas, J. N., Yates, J. R., Lafaille, J. J., et al. (2013). Microglia promote learning-dependent synapse formation through brain-derived neurotrophic factor. Cell 155, 1596-1609. doi: 10.1016/j.cell.2013. 11.030

Prinz, M., and Priller, J. (2014). Microglia and brain macrophages in the molecular age: from origin to neuropsychiatric disease. Nat. Rev. Neurosci. 15, 300-312. doi: $10.1038 / \mathrm{nrn} 3722$

Rockwood, D. N., Preda, R. C., Yücel, T., Wang, X., Lovett, M. L., and Kaplan, D. L. (2011). Materials fabrication from Bombyx mori silk fibroin. Nat. Protoc. 6, 1612-1631. doi: 10.1038/nprot.2011.379

Roy, J. (2018). Primary microglia isolation from mixed cell cultures of neonatal mouse brain tissue. Brain Res. 1689, 21-29. doi: 10.1016/j.brainres.2018.03.018

Schafer, D. P., Lehrman, E. K., Kautzman, A. G., Koyama, R., Mardinly, A. R., Yamasaki, R., et al. (2012). Microglia sculpt postnatal neural circuits in an activity and complement-dependent manner. Neuron 74, 691-705. doi: 10. 1016/j.neuron.2012.03.026

Stephan, A. H., Barres, B. A., and Stevens, B. (2012). The complement system: an unexpected role in synaptic pruning during development and disease. Annu. Rev. Neurosci. 35, 369-389. doi: 10.1146/annurev-neuro-061010-113810

Sun, W., Incitti, T., Migliaresi, C., Quattrone, A., Casarosa, S., and Motta, A. (2017). Viability and neuronal differentiation of neural stem cells encapsulated in silk fibroin hydrogel functionalized with an IKVAV peptide. J. Tissue Eng. Regen. Med. 11, 1532-1541. doi: 10.1002/term.2053 
Surinkaew, P., Sawaddiruk, P., Apaijai, N., Chattipakorn, N., and Chattipakorn, S. C. (2018). Role of microglia under cardiac and cerebral ischemia/reperfusion (I/R) injury. Metab. Brain Dis. 33, 1019-1030. doi: 10.1007/s11011-018-0232-4

Swistowski, A., Peng, J., Liu, Q., Mali, P., Rao, M. S., Cheng, L., et al. (2010). Efficient generation of functional dopaminergic neurons from human induced pluripotent stem cells under defined conditions. Stem Cells 28, 1893-1904. doi: 10.1002/stem.499

Tamashiro, T. T., Dalgard, C. L., and Byrnes, K. R. (2012). Primary microglia isolation from mixed glial cell cultures of neonatal rat brain tissue. J. Vis. Exp. 66:e3814. doi: 10.3791/3814

Tang-Schomer, M. D., White, J. D., Tien, L. W., Schmitt, L. I., Valentin, T. M., Graziano, D. J., et al. (2014). Bioengineered functional brain-like cortical tissue. Proc. Natl. Acad. Sci. U.S.A. 111, 13811-13816. doi: 10.1073/pnas.1324214111

van Pel, D. M., Harada, K., Song, D., Naus, C. C., and Sin, W. C. (2018). Modelling glioma invasion using 3D bioprinting and scaffold-free 3D culture. J. Cell. Commun. Signal. 12, 723-730. doi: 10.1007/s12079-018-0469-z

Weinhard, L., di Bartolomei, G., Bolasco, G., Machado, P., Schieber, N. L., Neniskyte, U., et al. (2018). Microglia remodel synapses by presynaptic trogocytosis and spine head filopodia induction. Nat. Commun. 9:1228. doi: 10.1038/s41467-018-03566-5

Yang, K., Lee, J., Lee, J. S., Kim, D., Chang, G. E., Seo, J., et al. (2016). Graphene oxide hierarchical patterns for the derivation of electrophysiologically functional neuron-like cells from human neural stem cells. ACS Appl. Mater. Interfaces 8, 17763-17774. doi: 10.1021/acsami.6b 01804

Conflict of Interest: The authors declare that the research was conducted in the absence of any commercial or financial relationships that could be construed as a potential conflict of interest.

Copyright (c) 2020 Zhu, Qiao, Liu, Wang and Zhao. This is an open-access article distributed under the terms of the Creative Commons Attribution License (CC BY). The use, distribution or reproduction in other forums is permitted, provided the original author(s) and the copyright owner(s) are credited and that the original publication in this journal is cited, in accordance with accepted academic practice. No use, distribution or reproduction is permitted which does not comply with these terms. 